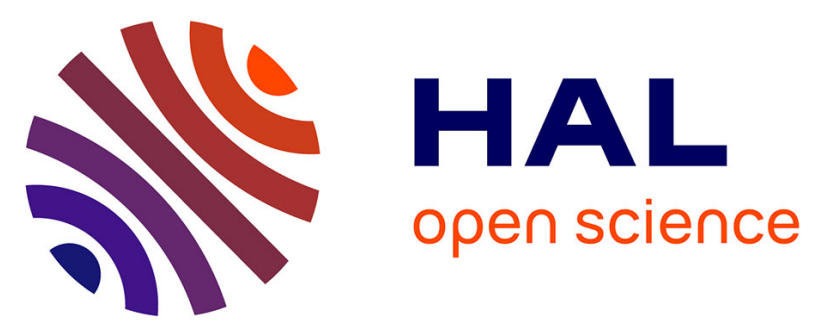

\title{
Optimisation of a sample preparation procedure for the screening of fungal infection and assessment of deoxynivalenol content on maize using mid-infrared attenuated total reflection spectroscopy
}

Gregor Kos, Hans Lohninger, Boris Mizaikoff, Rudolf Krska

\section{To cite this version:}

Gregor Kos, Hans Lohninger, Boris Mizaikoff, Rudolf Krska. Optimisation of a sample preparation procedure for the screening of fungal infection and assessment of deoxynivalenol content on maize using mid-infrared attenuated total reflection spectroscopy. Food Additives and Contaminants, 2007, 24 (07), pp.721-729. 10.1080/02652030601186111 . hal-00577546

\author{
HAL Id: hal-00577546 \\ https://hal.science/hal-00577546
}

Submitted on 17 Mar 2011

HAL is a multi-disciplinary open access archive for the deposit and dissemination of scientific research documents, whether they are published or not. The documents may come from teaching and research institutions in France or abroad, or from public or private research centers.
L'archive ouverte pluridisciplinaire HAL, est destinée au dépôt et à la diffusion de documents scientifiques de niveau recherche, publiés ou non, émanant des établissements d'enseignement et de recherche français ou étrangers, des laboratoires publics ou privés. 


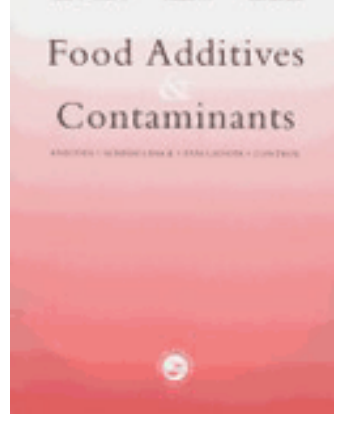

\section{Optimisation of a sample preparation procedure for the screening of fungal infection and assessment of deoxynivalenol content on maize using mid-infrared attenuated total reflection spectroscopy}

\begin{tabular}{|r|l|}
\hline Journal: & Food Additives and Contaminants \\
\hline Manuscript ID: & TFAC-2006-238.R1 \\
\hline Manuscript Type: & Original Research Paper \\
\hline Author: & 19-Dec-2006 \\
\hline Complete List of Authors: & $\begin{array}{l}\text { Kos, Gregor; University of Natural Resources and Applied Life } \\
\text { Sciences, IFA-Tulln, Center for Analytical Chemistry } \\
\text { Lohninger, Hans; Vienna University of Technology, Institute for } \\
\text { Chemical Technologies and Analytics } \\
\text { Mizaikoff, Boris; Georgia Institute of Technology, School of } \\
\text { Chemistry and Biochemistry } \\
\text { Krska, Rudolf; University of Natural Resources and Applied Life } \\
\text { Sciences, IFA-Tulln, Center for Analytical Chemistry }\end{array}$ \\
\hline Methods/Techniques: & $\begin{array}{l}\text { Mid Infrared Spectroscopy, Attenuated Total Reflection, } \\
\text { Chemometrics }\end{array}$ \\
\hline Additives/Contaminants: & Fusarium fungi, Mycotoxins \\
\hline Food Types: & Cereals and grain \\
\hline & \\
\hline
\end{tabular}

\section{SCHOLARONE" Manuscripts}


Optimisation of a sample preparation procedure for the screening of fungal infection and assessment of deoxynivalenol content of maize using mid-infrared attenuated total reflection spectroscopy 


\begin{abstract}
A sample preparation procedure for the determination of deoxynivalenol (DON) with mid-infrared spectroscopy is presented. Attenuated total reflection was employed with a mid-infrared spectrometer. Repeatable spectra were obtained from samples featuring a narrow particle size distribution. Samples were ground with a centrifugal mill and analysed with an analytical sieve shaker. Particle sizes of $<100 \mu \mathrm{m}, 100-250 \mu \mathrm{m}, 250-$ $500 \mu \mathrm{m}, 500-710 \mu \mathrm{m}$ and 710-1000 $\mu \mathrm{m}$ were obtained. Repeatability, classification and quantification abilities for DON were compared with non-sieved samples. The fraction "100-250 $\mu \mathrm{m}$ " showed the best repeatability: The relative standard deviation of spectral measurements improved from $20 \%$ to $4.4 \%$. $100 \%$ of sieved samples were correctly classified compared with $79 \%$ of non-sieved samples. The DON content in analysed fractions was a good estimate of the overall toxin content.
\end{abstract}

\title{
Keywords
}

attenuated total reflection, fungi, cereals, mycotoxins, sample preparation 


\section{Introduction}

The determination of fungal invasion of grain samples is of vital interest to food and feed producers as well as consumers alike. Food and feed that is contaminated by fungi may not only be unsuitable for food and feed processing, but can also be dangerous to human and animal health (Council for Agricultural Science and Technology 2003). Secondary fungal metabolites are often toxic and can cause serious health problems. In the moderate climatic zones of North America and Europe Fusarium fungi are among the most prevalent agriculturally important fungal species (Charmley et al. 1994). The main toxic secondary metabolite produced by these genera during the growth period of the host commodity is deoxynivalenol (DON), which causes feed refusal by swine, skin damage, vomiting and hemorrhages, if consumed (D'Mello et al. 1999).

Besides regulations for aflatoxins and ochratoxin A (EC 466/2001), maximum levels for Fusarium toxins in selected foodstuffs were recently set in the European Commission Regulation No 856/2005 which applies from July $1^{\text {st }} 2006$. Maximum levels for DON have been set e.g. at $700 \mu \mathrm{g} \mathrm{kg}^{-1}$ for cereal flour including maize flour. In the US, the Food and Drug Administration (FDA) has set advisory levels for DON to as low as $1000 \mu \mathrm{g} \mathrm{kg}^{-1}$ (Trucksess 1995).

The determination of DON itself (Krska et al. 2001) is well established, but tedious and labour intensive. Several sample preparation steps are necessary before separation and detection can take place using chromatographic methods such as LC-MS or GC-ECD. 
Determination could be sped up considerably with a rapid method. There is indeed a need for such a method, when considering the amounts of cereals that are processed by the food and feed industry each year. Food imports are subjected to frequent checks by authorities in Europe and the US and major entry points such as harbours and border crossings are facing an increased workload. A method for the determination of fungal contaminants within minutes is highly desirable in order to avoid long waiting times and increase the overall percentage of controlled goods.

So far, a small number of rapid methods have been proposed in order to assess the amount of fungal contamination in a given sample and not always the amount of toxic DON which is the more relevant parameter. Visual inspection is widely used for the assessment of maize cobs (Bechtel et al. 1985). The method is quick and cheap to perform, but labour-intensive and requires a lot of experience. DON levels are not directly estimated and the amount of fungus present on the cob is not always a reliable indicator for quantification, as it often resides inside the kernel (Greene et al. 1992). Samples that were already ground need more sophisticated investigation techniques, because a visual inspection is not possible.

The use of infrared spectroscopic techniques has already been proposed in previous studies as spectra can be easily recorded (Gordon et al. 1997; Dowell et al. 1999; Gourama and Bullerman 1995; Petterson and Aberg 2002). Delwiche and Gaines (2005) investigated visible and near-infrared (NIR) wavelengths for the determination of Fusarium head blight in winter wheat kernels. Near infrared instruments are popular 
and well-established in the analysis of foods and feeds, because the radiation penetrates through glass containers (Berardo et al. 2005). With Fourier Transform infrared (FTIR) instruments in the mid-infrared range, measurements can be performed very fast with an excellent signal to noise ratio and high-resolution power. In recent years several accessories have been developed that enable the measurements of solid samples without the need for extensive sample preparation (Harrick 1967). Photoacoustic infrared (IR-PAS) and diffuse reflection spectroscopies (IR-DR) have been employed for the detection of fungal species on maize and cereals (Greene et al 1992) and method performance was discussed previously (Kos et al. 2003; Kos et al. 2002). In these papers, the successful application of mid-infrared spectroscopy with attenuated total reflection (ATR) for the detection of fungal contamination on maize was described. The sample is placed on a horizontally mounted diamond ATR crystal and the mid infrared ATR spectrum is recorded. Maize was chosen as a commodity, because it is almost exclusively infected by Fusarium fungi during the growth period in moderate climates, thus minimizing contamination from other fungal species and making it a good model system to study.

The optimisation of the sample preparation procedure is a crucial point in obtaining reproducible and representative spectra. Cereal samples that are infected with fungal species have certain properties that make ATR measurements a challenging task with respect to repeatability of spectral measurements and prediction ability of the contaminant: 
The distribution of fungal contamination (and therefore its toxin) is not homogenous over the sample batch (Park and Pohland 1989). As consequence a small number of highly infected kernels can make a whole batch unsafe for food processing. The differences between the blank and the contaminated samples are small and usually not visible by the eye. PAS investigation of single kernels with high contamination levels revealed changes to the spectrum and in ATR spectra only highly contaminated samples show visible changes. Several spectral regions have to be excluded from calculations, because they either show high variability not related to fungal contamination (e.g. the $\mathrm{OH}$ moisture band and the ubiquitous, non-specific methylene bands)

Instrumental limitations can also cause the following problems:

After sample grinding a rather broad particle size distribution from $>1000 \mu \mathrm{m}$ to $<100 \mu \mathrm{m}$ in diameter is observed, which lead to repeatability problems in spectral measurements without any further pre-treatment (see Figure 1). Because of the small area of the ATR crystal, large sample particles may take up large amounts of space on the crystal, leading to over-representation in resulting spectra.

[Insert Figure 1 about here]

Large particles could also cause varying pressure conditions on the ATR crystal, when using a pressure applicator, which ensures full contact between the sample and the crystal. The range between 1800 and $2200 \mathrm{~cm}^{-1}$ is dominated by absorption of the diamond crystal and has to be removed from all datasets. 
This paper describes a sample preparation procedure that was developed in order to obtain representative samples that are suitable for ATR measurements and contain information about infection, which is accessible by mid infrared measurements. Results are compared to samples, which have not been treated, but were directly measured. The problems mentioned above are discussed and in order to overcome these problems sample preparation steps are presented.

\section{Materials and methods}

\section{Sample preparation}

All maize samples were chosen from the same genotype (RWA2) and were predominantly naturally infected with Fusarium graminearum in the field during the growth period. Thus, samples were as close as possible to real life samples, but still represented a very defined model system that eliminated any variability from parameters such as genotype variation and variation due to differing fungal species, providing a solid basis for the investigation of the repeatability of measurements.

After harvest, maize cobs were dried for 3 days by blowing air at $40^{\circ} \mathrm{C}$ through the storage container, similar to storage protocols used in commercial storage facilities. Thus, the moisture content was reduced to $<15 \%$, which stopped fungal growth (Schlegel 1993). After drying, whole kernels were separated from the cob and ground with a ZM 100 centrifugal mill (Retsch Haan, Germany) based on impact and shearing effects between a rotor and a fixed ring sieve. The sample kernels are pre-crushed on the 
wedge-shaped rotor teeth before further milling takes place between rotor and screen (see Figure 2).

[Insert Figure 2 about here]

Grinding of 50-100 $\mathrm{g}$ of sample was performed very quickly, usually in less than a min. The hole width of the ring sieve determined the upper particle size limit after grinding, which was collected in the cassette. Rotor speed was $14000 \mathrm{rpm}$. In this study a $1 \mathrm{~mm}$ stainless steel ring sieve was employed for all experiments.

\begin{abstract}
After grinding, parts of the sample were directly investigated with Fourier Transform Infrared (FTIR) Spectroscopy (see below) and for the second fraction (usually 30-50 g) the particle size distribution was determined by sieving with an analytical sieve shaker (Retsch AS200/Haan, Germany). Sieves with five different mesh sizes $(<100 \mu \mathrm{m}, 100$ $250 \mu \mathrm{m}, 250-500 \mu \mathrm{m}, 500-710 \mu \mathrm{m}$ and 710-1000 $\mu \mathrm{m})$ were employed. The sieving module was operated at an amplitude of $2 \mathrm{~mm}$ for a duration of $30 \mathrm{~min}$ using a 3dimensional throw movement. Two latex spheres were added to each sieve to minimise clogging. The particle size distribution was calculated prior to FTIR measurements.
\end{abstract}

\title{
Attenuated total reflection spectroscopy
}

All FTIR measurements were performed with a Bruker Vector 22 mid-infrared (MIR) spectrometer (Bruker Optics/Karlsruhe, Germany). A globar light source emitted broadband radiation that was coupled into a pendulum interferometer. After passing 
through the sample chamber, the radiation was detected with a L-alanine doted DTGS detector. The instrument is portable, because the chosen set-up requires no maintenance or service lines, making the spectrometer suitable for in-the field measurements.

Because of the unique properties of ATR spectroscopy for the easy measurement of solid samples, a horizontal ATR unit (SensIR-Technologies/Danbury, CT, US) was mounted in the sample chamber. It was fitted with a diamond ATR crystal with 3 internal reflections aligned to a ZnSe-crystal, which acted as a focusing element. The robustness and hardness of the crystal allowed for the use of a pressure applicator with a torque knob to ensure close contact between sample and crystal and the application of a reproducible pressure.

Spectra were collected at a resolution of $4 \mathrm{~cm}^{-1}$ and with a spectral range between 650 and $4500 \mathrm{~cm}^{-1}$. Background measurements were made against air by co-adding 32 scans for each spectrum. The interferometer was operated at a mirror speed of $10 \mathrm{kHz}$ and in the double-sided-bidirectional-mode. Fourier transformation was performed with a Mertz phase correction, a Blackman-Harris 3 term apodisation function and a zero filling factor of 2. No filters were applied. Each spectrum consisted of 1997 data points and was stored in a separate data file for further data processing.

\section{Reference methods}

In order to evaluate results from spectral measurements, especially for the estimation of 
differences between contaminated and blank samples, data from conventional methods for DON was obtained. Extraction, clean-up, separation and detection were performed with a method established using GC-ECD detection (Weingaertner et al. 1997): After extraction of ground wheat samples using an acetonitrile/water mixture, the filtered extracts were cleaned-up using Mycosep \#225 columns (Romerlabs, Tulln, Austria). After evaporation, the residue was derivatized using Sylon BTZ (Supelco, Vienna, Austria) and re-extracted into isooctance followed by analysis with a gas chromatograph with electron capture detection (GC-ECD) with Mirex as an internal standard. The limit of detection for DON in wheat was $65 \mu \mathrm{g} \mathrm{kg}^{-1}$. The mean recovery was $96.6 \%$ with a standard deviation of $9.5 \%$ for 47 samples spiked with DON. All samples were measured in a quality controlled environment.

Data analysis

Differences between spectra of blank and contaminated spectra are not easily detected, because of their high similarity. Therefore, it is necessary to reduce the variability of measurements that can not be related to fungal infection and additionally use the potential of multivariate data analysis for the detection of differences by employing several variables (i.e. wavelengths) for spectral calculations (Naes et al 2002).

All calculations were performed with Unscrambler® 7.5 (Camo/Oslo, Norway) and Datalab (H. Lohninger/Vienna, Austria) (Lohninger 2000). Repeatability calculations were carried out by the spectrometer software and in MS Excel (Microsoft/Redmond, OR, US). A minimum of 10 individual spectra was recorded from each sample, always 
using a new subsample. The fingerprint area (the range between 1800 and $800 \mathrm{~cm}^{-1}$ ) was chosen for all calculations. The final number of datapoints varied from 300-500 for each spectrum depending on the experiment. After appropriate data treatment (averaging, normalisation, derivative calculations) and principal component analysis (PCA) for the de-correlation of variables (Danzer et al. 2001), classification was performed using cluster analysis (CA). A quantification model was built with a partial least squares (PLS1) regression model. In order to assess the quality of the classification full cross validation was calculated, which is especially suited for the evaluation of small data sets, and the root mean square error of cross validation (RMSECV) determined (Naes et al. 2002). All results were cross-checked with trends obtained from raw data in order to detect artefacts caused by data treatment. A detailed description of the employed multi-variate methods can be found in an earlier publication (Kos 2003).

\section{Results and discussion}

The investigation of single kernels with ATR spectroscopy is theoretically possible, but not recommended. Because of the inhomogeneous distribution of fungus and toxin in a sample batch, a very large number of kernels would need investigation before any sound conclusions could be made for the whole batch and therefore sampling remains one of the central problems for representative mycotoxin analysis (Champeil et al. 2004). Therefore it is advisable to grind the sample in order to make it more representative of the lot (Garfield 1989).

In a first approach spectra were directly recorded from ground samples without any 
further sample preparation. Spectra for 10 repetitive measurements (each measured from a new sub-sample) are displayed in Figure 1. Band assignments are displayed in Table I. The rectangle marks the spectral range used for all calculations. The plots clearly illustrate the need for improvement, which is also reflected in a PCA score/score plot (Figure 3) and subsequent classification using cluster analysis (CA). Score plots already reveal a trend by the formation of two clusters, but correct classification of these clusters with CA is not possible (Figure 4): Contaminated samples with DON concentrations of 557, 1024 and $2596 \mu \mathrm{g} \mathrm{kg}^{-1}$ could not be classified correctly. Sample names in plots give DON concentrations in $\mu \mathrm{g} \mathrm{kg}^{-1}$ obtained from reference measurements. Individual blank measurements are marked with "bl_" and an identification number.

[Insert Table I about here]

[Insert Figure 3 about here]

[Insert Figure 4 about here]

The lack of repeatability could be due to the broad particle size distribution of the sample. A number of factors that might influence the repeatability of ATR measurements of particles that differ in diameter, when carrying out particle size measurements of silica particles have been described (Yoshidome et al. 1998). In order to overcome these limitations, the samples were subjected to particle size analysis.

The weight of fractions after sieving was determined and is displayed in Figure 5. The 
diagram also displays results for different contamination levels of DON in order to detect influences of the fungal contamination on the particle size distribution after grinding. The largest particle fraction had a particle size between 100 and $250 \mu \mathrm{m}$ in diameter, containing between 40 and $50 \%$ of the particles $(w / w)$. The fraction between 250 and $500 \mu \mathrm{m}$ contained an additional 20 to $30 \%$, which was still enough for a suitable number of repetitions for spectral measurements. The remaining fractions held only small amounts of material, usually not enough for ATR measurements.

The improvement in the repeatability of spectral measurements was estimated by calculating the relative standard deviation (RSD, \%) at five different wavelengths in the spectrum. From Figures in Table II it can be concluded that the repeatability of spectral measurements improves with a narrowing particle size distribution and smaller particle diameters. For the non-sieved sample a relative standard deviation (RSD) of almost $20 \%$ was calculated. This RSD decreased with the particle size reaching a low at $1.7 \%$ for the fraction with a particle diameter $<100 \mu \mathrm{m}$. From this point of view it was therefore obvious to choose the $250-100 \mu \mathrm{m}$ fraction for all subsequent measurements in this study, because it provided enough sample material for multiple repetitions (up to 15-25 g) and additional reference measurements and featured a satisfying repeatability of measurements $(4.4 \%)$. The smallest fraction $(<100 \mu \mathrm{m})$ did not yield enough sample in order to carry spectral measurements for all samples.

[Insert Table II about here] 
A score/score plot after PCA shows a good separation between the clusters of blank and contaminated samples (Figure 6), following the trend of the non-sieved sample. The dendrogram after CA (Figure 7) calculated from the first two principal components reflected this improvement and all samples could be assigned correctly into two classes (contaminated/not contaminated).

[Insert Figure 6 about here]

[Insert Figure 7 about here]

Five samples with their sieving fractions were chosen from all available samples and, if possible the DON content was determined in each of the fractions in order to assess, which fraction contained which amount of the reference analyte. Blank and contaminated samples with low (approx. 300 $\mu \mathrm{g} \mathrm{kg}^{-1}$ ), mid (approx. $1000 \mu \mathrm{g} \mathrm{kg}^{-1}$ ) and high (approx. $2600 \mu \mathrm{g} \mathrm{kg}^{-1}$ ) DON content were selected as a model system. All fractions that had enough sample left (2-10 g) after performing IR measurements were analysed for their DON content using the conventional procedure described above.

Table III summarises the results and illustrates that the DON content in the fraction chosen for ATR measurements gives a good estimate for the DON in the original (nonsieved) sample.

[Insert Table III about here]

\section{Conclusions and future work}


The results obtained here clearly illustrate the improvements that were achieved by applying particle size analysis to maize samples before ATR mid infrared measurements for the detection of fungal infection were carried out. Comparability is ensured, because all sub-samples in both datasets (not sieved and sieved) came from the same sample. The sample set was very defined (from a single maize genotype [RWA2] and fungal infection with $F$. graminearum), minimising variation from other sources than effects on spectral measurements by the particle size distribution.

By using sieved samples with a particle size fraction between 100 and $250 \mu \mathrm{m}$, it was possible to classify all 14 samples of the dataset in a concentration range of the reference analyte DON from $310 \mu \mathrm{g} \mathrm{kg}^{-1}$ to $2596 \mu \mathrm{g} \mathrm{kg}^{-1}$. With the dataset containing non-sieved samples only 11 out of 14 samples were assigned correctly. The relative standard deviation of spectral measurements could be reduced from almost $20 \%$ to $<5 \%$ for the chosen fraction, which is suitable and sufficient from an analytical point of view. A suitable size fraction has to be chosen after grinding or alternatively milling to the desired particle size has to be performed. It was shown for which particle sizes measurement variability is lowest, leading to stable and repeatable spectra. From reference measurements it was shown that the DON content in the investigated fraction is a good estimate of the overall DON content.

The current approach is not intended to be used directly in feed and food control, but rather demonstrates the effect of grinding and particle size for the determination of DON. The sieving procedure and the selection of a suitable particle size fraction 
eliminated a major source of unwanted variation in spectral measurements. Future work can therefore focus on the further optimisation of the ATR method. The investigation of additional sources that influence mid-infrared spectra is now possible. The (much smaller) variation caused by different maize genotypes and infection by several fungal species can now be investigated more easily, leading to a stable model for the estimation of the DON content.

\title{
Acknowledgements
}

This work was funded by the Austrian Science Fund (FWF, P-14096). The authors would like to thank Marc Lemmens/IFA-Tulln for providing the maize samples for this study.

\section{References}

Bechtel DB, Kaleikau LA, Gaines RL, Seitz LM. 1985. The effects of Fusarium graminearum infection on wheat kernels. Cereal Chemistry 62:191-197.

\author{
Berardo N, Pisacane V, Battiliane P, Scandolara A, Pietri A, Marocco A. 2005. Rapid \\ detection of kernel rots and mycotoxins in maize by near-infrared reflectance \\ spectroscopy. Journal of Agricultural Food Chemistry 53:8128-8134.
}

Champeil A, Fourbet J-F, Dore T. 2004. Effects of grain sampling procedures on Fusarium mycotoxin assays in wheat grains. Journal of Agricultural Food Chemistry $52: 6049-6054$. 
Charmley LL, Rosenberg A, Trenholm HL. 1994. Factors responsible for economic losses due to Fusarium mycotoxin contamination of grains, foods and feedstuffs. In: Miller JD, Trenholm HL, editors. Mycotoxins in Grain - Compounds Other Than Aflatoxins. St. Paul, MN: Eagan Press. p 471.

Council for Agricultural Science and Technology (2003): Mycotoxins and human disease. In: Mycotoxins - Risks in Plant, Animal and Human Systems, Ames. p 48.

Danzer K, Hobert K, Fischbacher C, Jagemann K-U. 2001. Chemometrik, Grundlagen und Anwendungen. Heidelberg: Springer. p 105.

Delwiche SR, Gaines CS. 2005. Wavelength selection for monochromatic and bichromatic sorting of Fusarium-damaged wheat. Applied Engineering in Agriculture 21:681-688.

D'Mello JPF, Placinta CM, Macdonald AMC. 1999. Fusarium mycotoxins: a review of global implications for animal health, welfare and productivity. Animal Feed Science and Technology 80:183-205.

Dowell FE, Ram MS, Seitz LM. 1999. Predicting scab, vomitoxin, and ergosterol in single wheat kernels using near infrared spectroscopy. Cereal Chemistry 76:573-576. 
Garfield FM. 1989. Sampling in the analytical scheme. Journal of the Association of Official Analytical Chemists 72:405-411.

Gordon SH, Schudy RB, Wheeler BC, Wicklow DT, Greene RV. 1997. Identification of fourier transform infrared photoacoustic spectral features for detection of aspergillus flavus infection in corn, International Journal of Food Microbiology 35:179-186.

Gourama H, Bullerman LB. 1995. Detection of molds in foods and feeds: Potential rapid and selective methods. Journal of Food Protection 58:1389-1394.

Greene RV, Gordon SH, Jackson MA, Bennett GA, McClelland JF, Jones RW. 1992. Detection of fungal contamination in corn - potential of FTIR-PAS and FTIR-DRS. Journal of Agricultural and Food Chemistry 40:1144-1149.

Harrick, NJ 1967. Internal Reflection Spectroscopy. New York: Wiley. p 12.

Kos G, Lohninger H, Krska R. 2002. Fourier transform mid-infrared spectroscopy with attenuated total reflection (FT-IR/ATR) as a tool for the detection of Fusarium fungi on maize. Vibrational Spectroscopy 29:115-119.

Kos G, Lohninger H, Krska R. 2003. Development of a method for the determination of Fusarium fungi on corn using mid-infrared spectroscopy with attenuated total reflection (FTIR-ATR) and chemometrics. Analytical Chemistry 75:1211-1217. 
Krska R, Baumgartner S, Josephs RD. 2001. The state-of-the-art in the analysis of typeA and -B trichothecene mycotoxins in cereals. Fresenius Journal of Analytical Chemistry 371:285-299.

Lohninger H. 2000. DataLab - A Program for Statistical Data Analysis Vers. 1.2. Available: http://www.lohninger.com/datalab.html. Accessed 2006 July 10.

Naes T, Isaksson T, Fearn T, Davies T. 2002. A user-friendly guide to multivariate calibration and classification. Chichester: NIR Publications. p 14.

Park DL, Pohland AE. 1989. Sampling and sample preparation for detection and quantitation of natural toxicants in food and feed. Journal of AOAC International 72:399-404.

Petterson H, Aberg L. 2002. Rapid estimation of deoxynivalenol and Fusarium by near infrared spectroscopy. Journal of Applied Genetics. 43A:141-144.

Trucksess, MW. 1995. Mycotoxins. Journal of AOAC International 78:135-141.

Schlegel HG. 1993. General Microbiology. Cambridge: Press Syndicate of the University of Cambridge. p 199. 


\author{
Weingaertner J, Krska R, Praznik W, Grasserbauer M, Lew H. 1997. Use of mycosep \\ multifunctional clean-up columns for the determination of trichothecenes in wheat by \\ electron capture gas chromatography. Fresenius Journal of Analytical Chemistry \\ $357: 1206-1210$.
}

Yoshidome T, Kusumoto H, Kuroki O, Kamata S. 1998. Application of the attenuated total reflection technique to the measurement of silica gel particle size. Chemistry Letters 8:747-748. 


\section{Tables}

Table I. Band assignments for a mid-infrared ATR spectrum of maize.

Table II. Relative standard deviation [\%] of spectral measurements from a single sample. For each fraction datapoints were taken from 5 different wavelengths and 10 repetitions of the ATR measurement, each of which was performed with a new subsample.

Table III. DON reference data $\left[\mu \mathrm{g} \mathrm{kg}^{-1}\right]$ for particle size fractions. Detection limit of the reference method was $65 \mu \mathrm{g} \mathrm{kg}^{-1}$. (n.d. - not detected, n.m. - not measured, lack of sample). 


\section{Figures}

Figure 1. Repeatability of mid-infrared ATR spectra of maize $(n=10)$. The sample was ground only, without any further pre-treatment. Band assignments are displayed in Table I. The rectangle marks the spectral range used for all calculations.

Figure 2. Set-up of the ZM 100 centrifugal mill (view from top). (1) feed, (2) rotor teeth, (3) fixed ring sieve, (4) collector, (5) mill housing.

Figure 3. PCA of averaged ATR spectra that were not sieved before spectral measurements. Sample numbers in plot give DON concentrations in $\left[\mu \mathrm{g} \mathrm{kg}^{-1}\right]$. Each datapoint was averaged from 10 repetitive measurements.

Figure 4. Cluster Analysis of the first 2 principal components calculated from the PCA results obtained from Figure 3 (samples not sieved).

Figure 5. Particle size distribution after sieving of ground maize samples. Different levels of fungal contamination (expressed as DON content) were investigated for their behaviour during the grinding process.

Figure 6. PCA of averaged ATR spectra that were sieved before spectral measurements. Particle size fraction was between 250 and $100 \mu \mathrm{m}$. Sample numbers in plot give DON concentrations in $\left[\mu \mathrm{g} \mathrm{kg}^{-1}\right]$. Each datapoint was averaged from 10 repetitive 
measurements.

Figure 7. CA of the first 2 principal components calculated from the PCA results obtained from Figure 6 (sieved samples). 
Table I. Band assignments for a mid-infrared ATR spectrum of maize.

\begin{tabular}{llll}
\hline Wavenumber $\left[\mathrm{cm}^{-1}\right]$ & No in fig. 2 & Assignment of Bands & Relative Intensity \\
\hline 3300 & $(1)$ & Hydroxyl stretching & Strong \\
2930,2855 & $(2)$ & Methylene stretching & Strong \\
$2325-1900$ & $(3)$ & Diamond crystal absorptions & Strong \\
1745 & $(4)$ & C=O stretching & Weak \\
1540 & $(5)$ & NH bending/CN stretching & Weak \\
990 & $(6)$ & C-O stretching & Strong \\
\hline
\end{tabular}


Table II. Relative standard deviation [\%] of spectral measurements from a single sample. For each fraction datapoints were taken from 5 different wavelengths and 10 repetitions of the ATR measurement, each of which was performed with a new subsample.

\begin{tabular}{cccrrrr}
\hline \multicolumn{7}{c}{ Fraction: Particle size $[\mu \mathrm{m}]$} \\
Wavelength $\left[\mathrm{cm}^{-1}\right]$ & Not sieved & $>710$ & $710-500$ & $500-250$ & $250-100$ & $<100$ \\
\hline 815.8 & 18.4 & 7.05 & 6.93 & 3.37 & 3.42 & 1.33 \\
997.1 & 20.0 & 23.1 & 25.4 & 13.6 & 4.12 & 4.71 \\
1336 & 18.8 & 5.52 & 5.79 & 2.86 & 3.61 & 0.85 \\
1643 & 20.4 & 6.81 & 6.68 & 2.22 & 3.61 & 1.34 \\
1839 & 21.8 & 0.92 & 0.72 & 0.47 & 7.08 & 0.21 \\
Mean & $\mathbf{1 9 . 9}$ & $\mathbf{8 . 6 8}$ & $\mathbf{9 . 1 0}$ & $\mathbf{4 . 4 9}$ & $\mathbf{4 . 3 7}$ & $\mathbf{1 . 6 9}$ \\
\hline
\end{tabular}


Table III. DON reference data $\left[\mu \mathrm{g} \mathrm{kg}^{-1}\right]$ for particle size fractions. Detection limit of the reference method was $65 \mu \mathrm{g} \mathrm{kg}^{-1}$. (n.d. - not detected, n.m. - not measured, lack of sample).

\begin{tabular}{lcccccc}
\hline \multicolumn{7}{l}{ Fraction: Particle size $[\mu \mathrm{m}]$} \\
Sample & Original & $>710$ & $710-500$ & $500-250$ & $250-100$ & $<100$ \\
\hline b1_2 & n.d. & n.d. & n.d. & n.d. & n.d. & n.d \\
b1_5 & n.d. & n.d. & n.d. & n.d. & n.d. & 140 \\
309 & 309 & 375 & 488 & 380 & 263 & n.m. \\
1173 & 1173 & n.m. & 1290 & 1179 & 1185 & n.m. \\
2596 & 2596 & 1979 & 2251 & 3452 & 4529 & n.m. \\
\hline
\end{tabular}




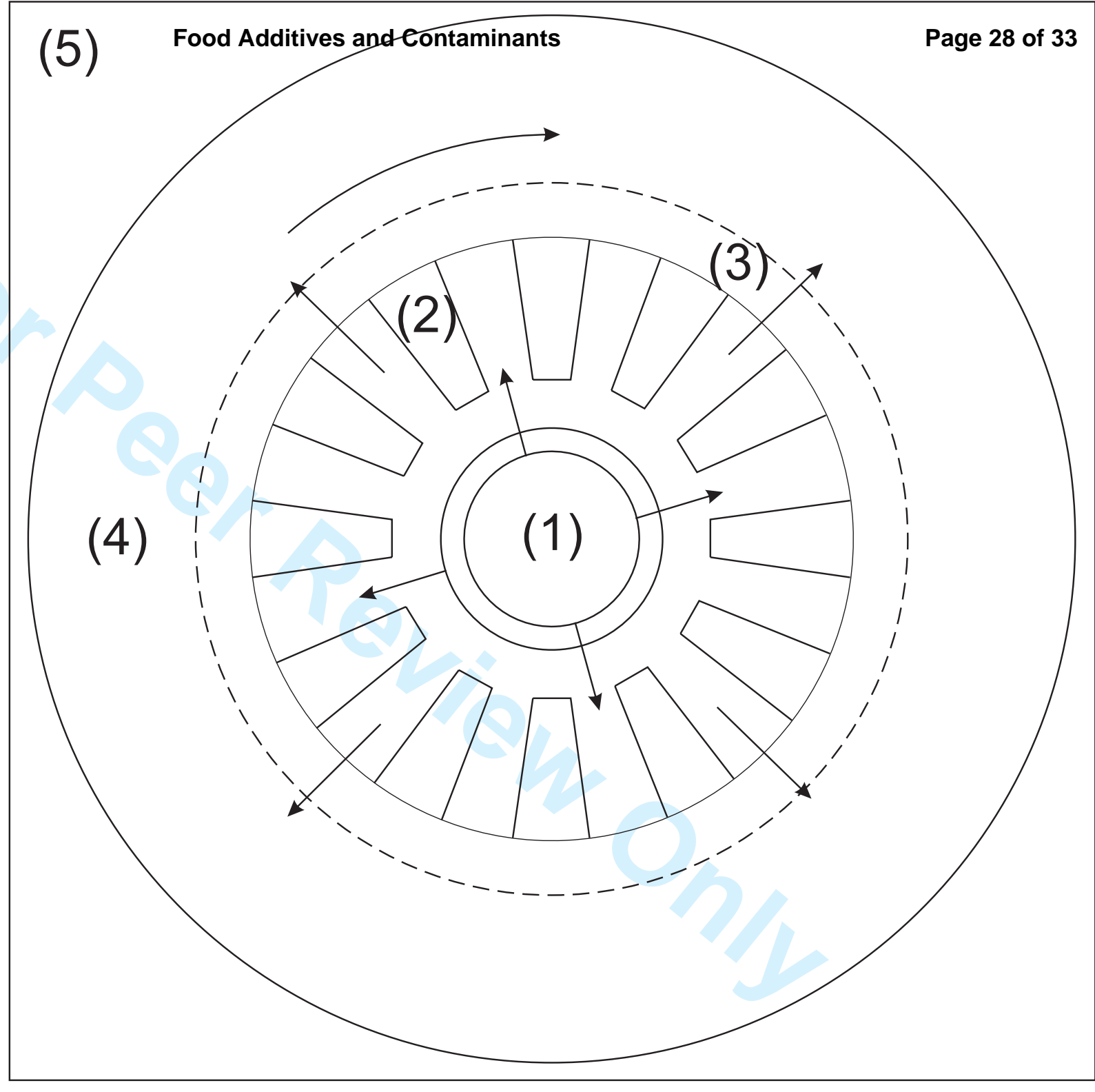

http://mc.manuscriptcentral.com/tfac Email: fac@tandf.co.uk 


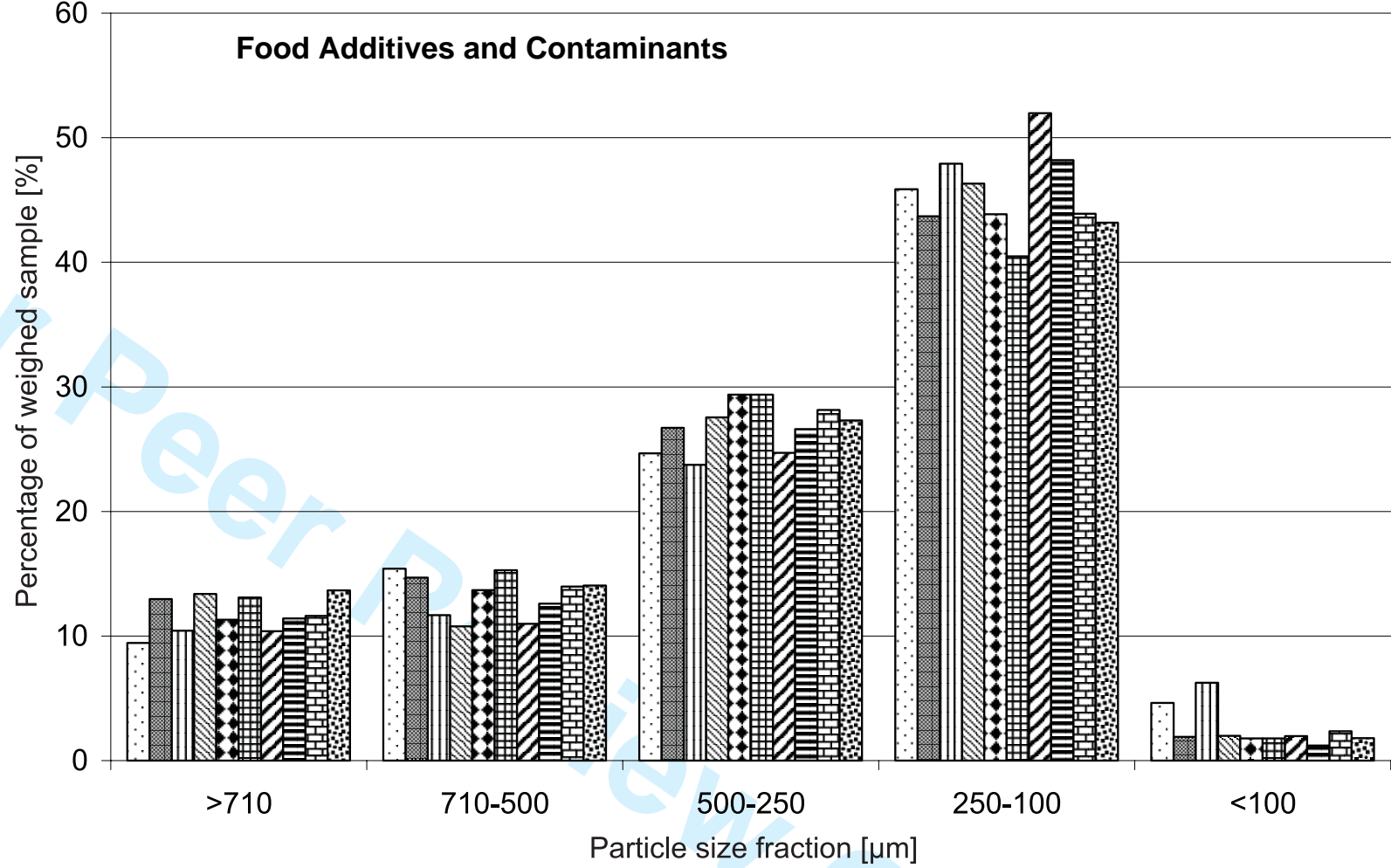

21

22

23 


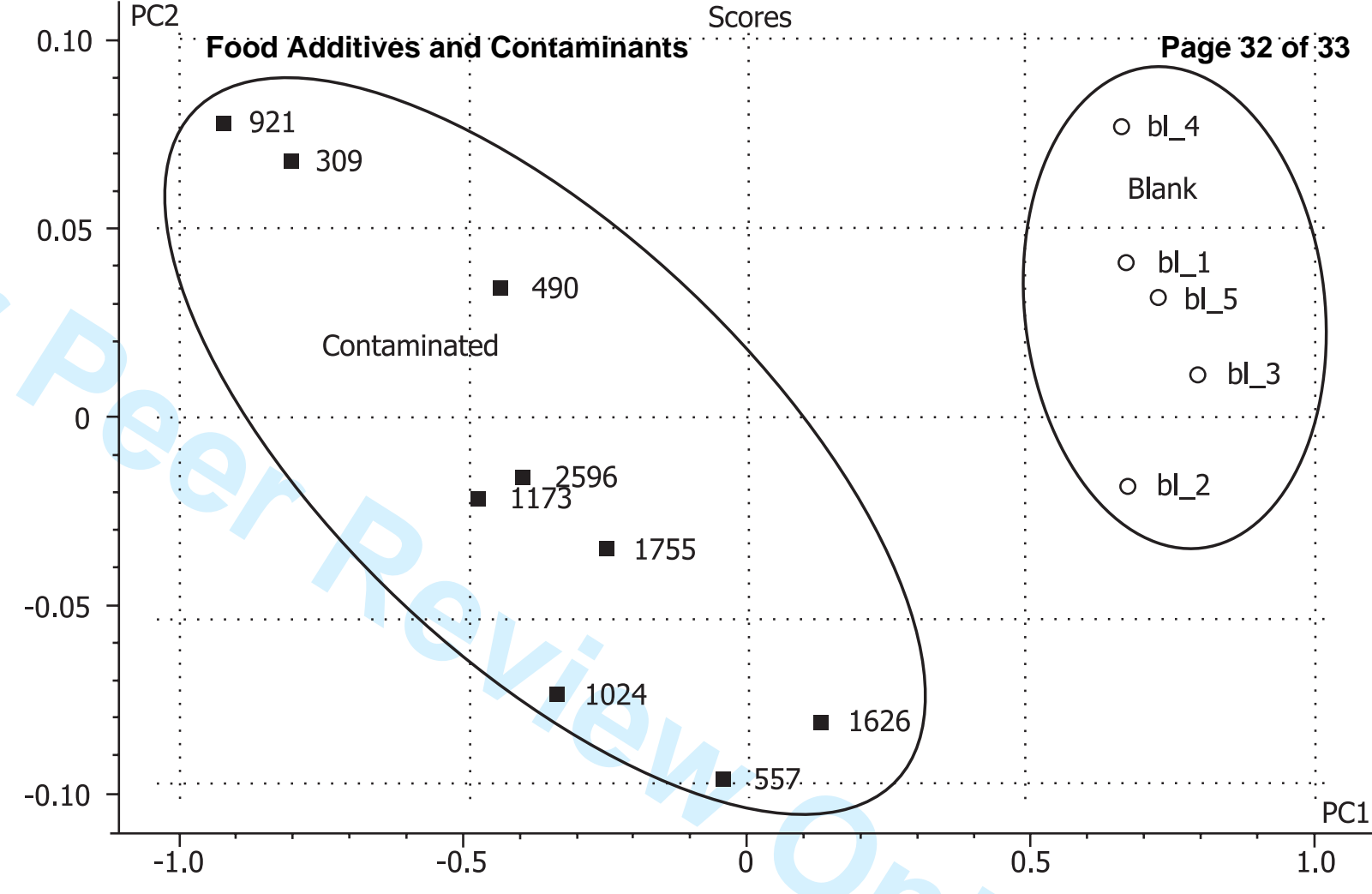

http://mc.manuscriptcentral.com/tfac Email: fac@tandf.co.uk 


\section{Page 33 of 33}

Food-Additives and Contaminants

1

2

3

4

5

6

7

8

10

11

12

13

14

15

16

17

18

19

20

21

22

23

24

25

26

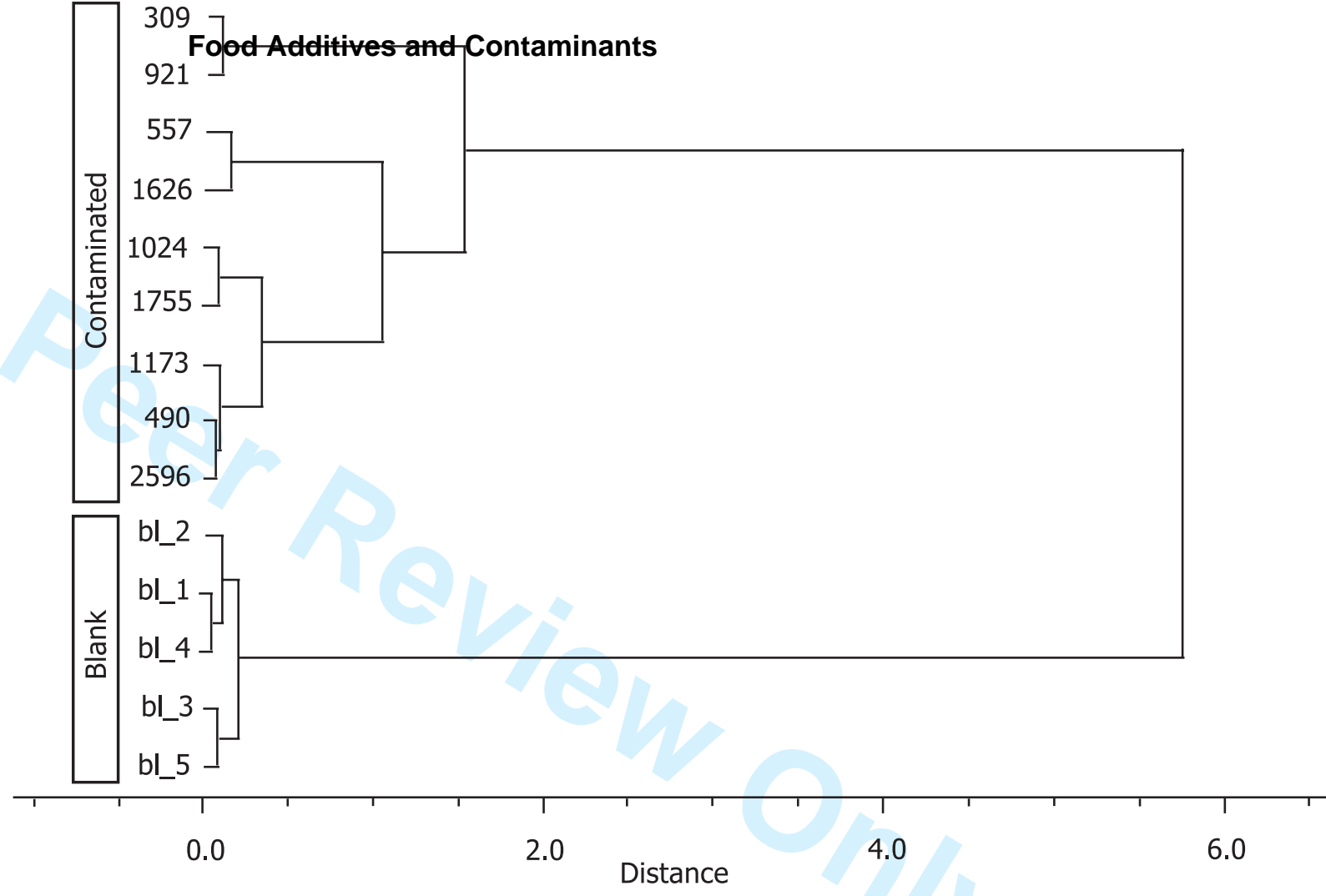

